\title{
Laplace Transform of Spherical Bessel Functions
}

\author{
A. Ludu \\ Department of Chemistry and Physics, \\ Northwestern State University, Natchitoches, LA 71497 \\ R. F. O'Connell \\ Department of Physics and Astronomy, \\ Louisiana State University, Baton Rouge, LA 70803-4001
}

\begin{abstract}
We provide a simple analytic formula in terms of elementary functions for the Laplace transform $\tilde{j}_{l}(p)$ of the spherical Bessel function than that appearing in the literature, and we show that any such integral transform is a polynomial of order $l$ in the variable $p$ with constant coefficients for the first $l-1$ powers, and with an inverse tangent function of argument $1 / p$ as the coefficient of the power $l$. We apply this formula for the Laplace transform of the memory function related to the Langevin equation in a one-dimensional Debye model.
\end{abstract}

PACS numbers: 02.30.Gp, 02.30.Uu

${ }^{1}$ E-mail address: ludua@nsula.edu

${ }^{2}$ E-mail address: phrfoc@lsu.edu 


\section{Introduction. The memory function in the Debye model.}

In recent years, there has been widespread interest in dissipative problems arising in a variety of areas in physics, and we refer to [1] for a mini-review. As it turns out, solutions of many of these problems are encompassed by a generalization of Langevin's equation to encompass quantum, memory and non-Markovian effects, as well as arbitrary temperature and the presence of an external potential $\mathrm{V}(\mathrm{x})$. As in [2], we refer to this as the generalized quantum Langevin equation (GLE):

$$
m \ddot{x}+\int_{-\infty}^{t} d t^{\prime} \mu\left(t-t^{\prime}\right) \dot{x}\left(t^{\prime}\right)+V^{\prime}(x)=F(t)+f(t),
$$

where $V^{\prime}(x)=d V(x) / d x$ is the negative of the time-independent external force and $\mu(t)$ is the so-called memory function. $F(t)$ is the random (fluctuation or noise) force and $f(t)$ is a c-number external force (due to a gravitational wave, for instance). In addition (keeping in mind that measurements of $\Delta x$ generally involve a variety of readout systems involving electrical measurements), it should be strongly emphasized that "- the description is more general than the language -" [2] in that $x(t)$ can be a generalized displacement operator (so that, for instance, $\Delta x$ could represent a voltage change). Furthermore, $\mu(t)$ and $F(t)$ are given in terms of the parameters of the heat bath only. Explicitly

$$
\mu(t)=\sum_{j} m_{j} \omega_{j}^{2} \cos \left(\omega_{j} t\right) \theta(t)
$$

where $\theta(t)$ is the Heaviside step function. Also

$$
F(t)=\sum_{j} m_{j} \omega_{j}^{2} q_{j}^{h}(t)
$$

where $q^{h}(t)$ denotes the general solution of the homogeneous equation for the heatbath oscillators (corresponding to no interaction). Thus, we have all the tools necessary for the analysis of any heat bath. As emphasized in Refs. [1,3] of primary interest for the calculation of observable physical quantities are either the Fourier or Laplace transforms of $\mu(t)$. In particular, the blackbody radiation heat bath was investigated in detail in Refs. $[2,3]$.

Here, we wish to consider the one-dimensional Debye model [4] because it leads to a result for the memory function which involves spherical Bessel functions.

In this model one takes all the heat bath oscillators to have the same mass i.e. $m_{j} \equiv m$ for all $j$. Thus, taking the maximum allowed heat bath frequency to be $\omega_{L}$ we see that Eq. (2) reduces to

$$
\mu(t)=m \sum_{j} \omega_{j}^{2} \cos \omega_{j} t=m \int_{0}^{\omega_{L}} d \omega D(\omega) \omega^{2} \cos \omega t
$$


where [4]

$$
D(\omega)=\frac{L}{\pi v}
$$

is the density-of-states. Also, $v$ is the velocity of sound and $L$ is the length of a one-dimensional line such that $\omega=v k$ where $\Delta k=(2 \pi / L)$ is the interval between the allowed values of the wave-vector $k$. It follows that

$$
\mu(t)=\frac{m L}{\pi v} \int_{0}^{\omega_{L}} d \omega \omega^{2} \cos \omega t=\frac{m L}{3 \pi v} \omega_{L}^{3}\left\{j_{0}\left(\omega_{L} t\right)-2 j_{2}\left(\omega_{L} t\right)\right\}
$$

Thus, in order to calculate $\tilde{\mu}(p)$ we will turn to our general result given in the next section and related to Laplace transform of spherical Bessel functions. For example, for the above case, the following expression for the Laplace is obtained

$$
\tilde{\mu}(p)=\frac{m L}{\pi v} \omega_{L} p-p^{2} \tan ^{-1}\left(\frac{\omega_{L}}{p}\right), .
$$

from a more general formula. For a detailed discussion of this result we refer to Ref.[5].

\section{Analytic formula for the Laplace transform.}

In order to calculate eq.(7), as well as the Laplace transform of higher order Bessel functions for other applications, we introduce in the following an exact formula, much simpler than formulas found in literature, in terms of trigonometric functions and polynomials.

The spherical Bessel functions are given by (Ref. 6, p. 965)

$$
j_{l}(t)=\left(\frac{\pi}{2 t}\right)^{\frac{1}{2}} J_{l+\frac{1}{2}}(t)
$$

where $l$ is a positive integer and $J_{\nu}$ are the Bessel functions of the first kind of real argument $t$. The Laplace transform, defined by

$$
L\left[j_{l}(t)\right] \equiv \tilde{j}_{l}(p)=\int_{0}^{\infty} j_{l}(t) e^{-p t} d t
$$

can be calculated by using the relation [Ref. 7, p. 182, II (9)]

$$
L\left[t^{\mu} J_{\nu}(t)\right]=\Gamma(\mu+\nu+1) \frac{P_{\mu}^{-\nu}\left(\frac{p}{\sqrt{p^{2}+1}}\right)}{\left(p^{2}+1\right)^{\frac{\mu+1}{2}}},
$$


where $P_{\mu}^{\nu}$ are the Legendre functions and we have to fulfill the restrictions: $\mu+\nu>-1$ and $p>0$. Also, $\Gamma$ is the Gamma function defined [6] as $\Gamma(x)=\int_{0}^{\infty} e^{-t} t^{x-1} d t$ for positive values of $x$.

In the case of the spherical Bessel functions we have $\nu=l+1 / 2$ and $\mu=-1 / 2$ and hence the restriction is $l>-1$ which is always fulfilled since $l=0,1, \ldots$ Hence

$$
\begin{gathered}
\tilde{j}_{l}(p)=\sqrt{\frac{\pi}{2}} \Gamma(l+1)\left(\frac{\rho}{p}\right)^{\frac{1}{2}} P_{-\frac{1}{2}}^{-l-\frac{1}{2}}(\rho) \\
=\sqrt{\frac{\pi}{2}} \frac{\Gamma(l+1)}{\Gamma(l+3 / 2)}\left(\frac{\rho}{p}\right)^{\frac{1}{2}}\left(\frac{1-\rho}{1+\rho}\right)^{\frac{l+\frac{1}{2}}{2}} F\left(\frac{1}{2}, \frac{1}{2} ; l+\frac{3}{2} ; \frac{1-\rho}{2}\right),
\end{gathered}
$$

where $\rho=p / \sqrt{p^{2}+1}$ and $p>0$ so that $1>\rho>0$. Here $F(\alpha, \beta ; \gamma ; x)$ is the Gauss hypergeometric series defined by the formula [6]

$$
F(\alpha, \beta ; \gamma ; x)=\sum_{k=0}^{\infty} \frac{\alpha^{(k)} \beta^{(k)}}{\gamma^{(k)}} \cdot \frac{z^{k}}{k !},
$$

where $\alpha^{(k)}=\alpha(\alpha+1) \ldots(\alpha+k-1) ; \alpha^{(0)}=1$.

The formula provided by eq.(11) is somehow difficult to use in the cases $l>2$, and requires tedious calculations and further manipulations such as integration or other transforms. However, in the case $l=0$, eq.(11) reduces to

$$
\tilde{j}_{0}(p)=\sqrt{2}\left(\frac{\rho}{p}\right)^{\frac{1}{2}}\left(\frac{1+\rho}{1-\rho}\right)^{-\frac{1}{4}} F\left(\frac{1}{2}, \frac{1}{2} ; \frac{3}{2} ; \frac{1-\rho}{2}\right) .
$$

Since the Gauss hypergeometric series in eq.(12) can be writen in the form (Ref. 6, p. 1041 II 13)

$$
F\left(\frac{1}{2}, \frac{1}{2} ; \frac{3}{2} ; \rho\right)=\frac{\sin ^{-1} \sqrt{\rho}}{\sqrt{\rho}},
$$

we find for the Laplace transform of $j_{0}(t)=\sin t / t$ the usual expression (Ref. 7, p. 152)

$$
\tilde{j}_{0}=\tan ^{-1} \frac{1}{p} .
$$

Another possibility for expressing the Legendre function for the $l=0$ case in a simpler way is to use the result ( Ref. 6, p. 1008)

$$
P_{-\frac{1}{2}}^{-\frac{1}{2}}(\rho)=\lim _{q \rightarrow 0} P_{q-\frac{1}{2}}^{-\frac{1}{2}}(\rho)=\cos ^{-1}(\rho) \sqrt{\frac{2}{\pi \sqrt{1-\rho^{2}}}} .
$$

Finally, one can use a direct approach, based on the fact that the Fourier representation of the spherical Bessel functions are definite integrals over Legendre poynomials

$$
j_{l}(t)=\frac{1}{2}(-i)^{l} \int_{-1}^{1} e^{i l x} P_{l}(x) d x .
$$


The following step is to perform the integration for the Laplace transform, obtaining the relation $\tilde{j}_{l}(p)=i^{l+1} Q_{l}(i p)$, where $Q_{l}(p)$ are the Legendre functions of second type [6-8].

However, none of these approaches can provide an analytic expression in terms of elementary functions for the $l \geq 2$ case. Thus, we are motivated to develop a new approach based on recursion relations. By differentiating two times the second order spherical Bessel function

$$
j_{2}=\left(\frac{3}{t^{2}}-1\right) \frac{\sin t}{t}-\frac{2 \cos t}{t^{2}},
$$

and by using recurrently the formula for the Laplace transform of the derivative of a function $f(t)$ (Ref. 7, p. 129)

$$
\tilde{f}^{\prime}(p)=p \tilde{f}(p)-f(0),
$$

where the prime denotes the derivative with respect to $p$, we can calculate the Laplace transform of $j_{2}$. That is we choose for $f(t)=\sin \alpha t / t$ which has its Laplace transform in tables $[6,7]$. We differentiate $f(t)$ once and we calculate the Laplace transform of $f^{\prime}(t)$ by using Eq.(17). Then, we differentiate one more time and find again the Laplace transform of $f^{\prime \prime}(x)$ by formula Eq.(17). Since we can express $j_{2}$ only in terms of the functions $f(t)$ and $f^{\prime \prime}(x)$ we can express its Laplace transform in terms of the Laplace transforms of $f(t), f^{\prime \prime}(t)$. We finally obtain

$$
\tilde{j}_{2}=\left(\frac{3 p^{2}}{2}+\frac{1}{2}\right) \tan ^{-1} \frac{1}{p}-\frac{3 p}{2} .
$$

This procedure gives us a hint for constructing a recursion formula for the Laplace transform of the spherical Bessel functions of any order. This will provide a much simpler form than that one provided by eq.(11). This approach needs only the derivative recursion formula, eq.(17), and the transform of the zero order function. By starting from the recursion formulas for the spherical Bessel functions (Ref. 6, p. 967)

$$
\begin{gathered}
j_{l+1}(\rho)=-j_{l}^{\prime}(\rho)+\frac{l}{\rho} j_{l}(\rho) \\
j_{l+1}(\rho)=\frac{2 l+1}{\rho} j_{l}(\rho)-j_{l-1}(\rho), \quad l \geq 1,
\end{gathered}
$$

and by writing them in a convenient form

$$
j_{l+1}=-\frac{2 l+1}{l+1} j_{l}^{\prime}+\frac{l}{l+1} j_{l-1},
$$

we find that any spherical Bessel function can be expressed as a sum of the derivatives of different orders of $j_{0}$

$$
j_{l}(\rho)=\sum_{k=0}^{2 k \leq l} C_{k}^{(l)} \frac{d^{l-2 k}}{d \rho^{l-2 k}} j_{0}(\rho),
$$


where $C_{k}^{(l)}$ are coefficients depending on the labels $l$ and $k$. In order to calculate these coefficients we use again the recursion relation eq.(20) and the formula in eq.(21) and we obtain the equation

$$
\sum_{k=0}^{2 k \leq l} C_{k}^{(l)} j_{0}^{(l-2 k+1)}=\frac{l}{2 l+1} \sum_{k=0}^{2 k \leq l-1} C_{k}^{(l-1)} j_{0}^{(l-2 k-1)}-\frac{l+1}{2 l+1} \sum_{k=0}^{2 k \leq l+1} C_{k}^{(l+1)} j_{0}^{(l-2 k+1)},
$$

where the superscript on the Bessel function indicates the order of differentiation. Since the Wronskian form of any two derivatives of $j_{0}$ is not identical to zero, all these derivatives are functional independent so we can determine the numbers $C_{k}^{(l)}$ in eq.(22) by indentifying the coefficients of the same order of derivative of the Bessel function. Consequently we find the binary recursion relation

$$
C_{0}^{l+1}=-\frac{2 l+1}{l+1} C_{0}^{(l)}
$$

which provide all $k=0$ coefficients

$$
C_{0}^{(l+1)}=(-1)^{l+1} \frac{(2 l+1) ! !}{(l+1) !}, \quad l=0,1, \ldots \text { and } C_{0}^{(0)}=0 .
$$

and a ternary recursion relation

$$
C_{k}^{(l+1)}=-\frac{2 l+1}{l+1} C_{k}^{(l)}+\frac{l}{l+1} C_{k-1}^{(l-1)}, \quad l=1,2, \ldots, \quad k \leq l,
$$

which provides the other coefficients. Also, if we are given $C_{1}^{(2)}$ (which can be easily obtained by giving particular values in eq.(22), that is $C_{1}^{(2)}=1 / 2$ ) we can generate all the coefficients by using the ternary relation, eq.(25). By using this procedure it is easy to identify all coefficients in the formula eq.(21). For example we have

$$
\begin{gathered}
j_{1}=-j_{0}^{(1)}, \quad j_{2}=\frac{3}{2} j_{0}^{(2)}+\frac{1}{2} j_{0}, \quad j_{3}=-\frac{5}{2} j_{0}^{(3)}-\frac{3}{2} j_{0}^{(1)}, \\
j_{4}=\frac{35}{8} j_{0}^{(4)}+\frac{15}{4} j_{0}^{(2)}+\frac{3}{8} j_{0}, \quad \text { etc. }
\end{gathered}
$$

The next step is to calculate the Laplace transform by using eqs.(17) and (21). Since

$$
j_{0}^{(2 k)}(0)=\frac{(-1)^{k}}{2 k+1}, \quad j_{0}^{(2 k+1)}(0)=0,
$$

and from eqs.(14) and (17), we have

$$
L\left[j_{0}^{(n)}\right]=p^{n} \tan ^{-1} \frac{1}{p}-\sum_{m=0}^{n-1} p^{n-m-1} j_{0}^{(m)}(0),
$$


we finally obtain the desired formula

$$
\tilde{j}_{l}(p)=p^{l}\left[\tan ^{-1} \frac{1}{p} \cdot \sum_{k=0}^{2 k \leq l} C_{k}^{(l)} p^{-2 k}-\sum_{k=0}^{2 k \leq l} C_{k}^{(l)} \sum_{m=0}^{\left[\frac{l-1}{2}\right]-k} \frac{(-1)^{m}}{2 m+1} p^{-2 k-2 m-1}\right],
$$

valid for $l \geq 1$, where [ · ] stands for the integer part. The Laplace transform is hence given in terms of elementary functions, by a polynomial $Q$ of order $l-1$ in the variable $p$, with constant coefficients (all coefficients determined by the recursion relations eqs.(24) and (25)) plus another polynomial $P$ of order $l$ multiplied by the inverse tangent function of $1 / p$

$$
\tilde{j}_{l}=P_{l}(p) \tan ^{-1}(1 / p)+Q_{l-1}(p) .
$$

Finally, we compare the above algorithm, based on recurrsion relations Eqs.(24, 25) and Eqs. (29, 30), with direct numerical algorithms used in the corresponding built-in functions in different symbolic programs, like for instance Mathematica. All functions involved above have their equivalent built-in correspondent in Mathematica-4.0 (used on a Power Macintosh 8500 computer), that is Gamma[z], BesselJ[n,z], LegendreP[n,z], Legendre $Q[n, m, z]$, etc. While comparing the CPU time elapsed for both procedures we find out that our recurrsion approach is faster. That is the relative difference between the two CPU intervals of time increases as $e^{3.2 l}$ (numerical with respect to analytic), where $l$ is the order of the spherical Bessel function.

\section{Conclusions}

By using a generalization of the quantum Langevin's equation in terms of memory function one can obtain exact solutions for dissipative problems arising in many areas in physics. In terms of a one-dimensional Debye model the memory function is expressed as a combination of spherical Bessel functions. However, of primary interest for the claculation of physical quantities are the laplace (or Fourier) transform of the memory function.

Also, the Schrödinger equation for a free particle in polar coordinates leads, for each value of the positive integer $l$ of the orbital angular momentum, to a radial equation which results in the generic equation for spherical Bessel functions. In electrodynamics, too, the spherical Bessel functions are related to solutions of the field equations in the stationary or quasi-stationary regime in cylindrical geometry. And moreover, Bessel functions are involved in the Helmholtz equation in cylindrical coordinates. In all such 
applications one needs the integral transforms (especially the Fourier or Laplace transforms) of such solutions. Constructing an exact analytic formula for such applications is the object of our studyy. Consequently, the present letter is not addressed to experts in special functions, but rather to physicists who just need to apply such formulas in their research, in the simplest possible form.

We have obtained a simpler exact formula for the Laplace transform of the Spherical Bessel functions of any order, in terms of polynomial and trigonometric functions, that is in terms of elementary functions. Among other representations of such functions, this expression is much simpler, hence more useful for potential applications. The speed of calculation is one of foremost importance for applications similar with the one presented above, especially in more than one dimension. We compared this formula with some numerical evaluation in terms of precision and suitability for faster implementation. Our comparison shows that the introduced formula is easy to be used and it introduces less computational complexity than conventional numerical techniques.

\section{Aknowledgments}

The authors are thankful to P. Abbott, The University of Western Australia, for his valuable comments.

\section{References}

[1] R. F. O'Connell, "Dissipative and Fluctuation Phenomena in Quantum Mechanics with Application," in Festschrift for J. P. Dahl, Intr. J. Quantum Chem., 58, 569 (1996).

[2] G. W. Ford, J. T. Levis and R. F. O'Connell, Phys. Rev. A 37, 44193 (1998).

[3] G. W. Ford, J. T. Levis and R. F. O'Connell, Phys. Rev. Lett. 55, 2273 (1985).

[4] C. Kittel, "Introduction to Solid State Physics," $7^{\text {th }}$ edition (J. Wiley, New York, 1996), pps. 118-120. 
[5] J. Kim and M. H. Lee, to be published. The authors have obtained a similar formula using the method of recurrence relations, which turns out to be much more complicated for the purpose of this calculations.

[6] I. M. Ryshik and I. S. Gradstein, Tables of Series, Products and Integrals, $4^{\text {th }}$ edition (Academic press, New York, 1980).

[7] H. Bateman, Tables of Integral Transforms Vol. I (McGraw-Hill Book Company, Inc., New York, 1954).

[8] M. Abramowitz and I. Stegun, Handbook of Mathematical Functions (Dover, 1970). 